\title{
Proof of an intersection theorem via graph homomorphisms.
}

\author{
Irit Dinur * $\quad$ Ehud Friedgut ${ }^{\dagger}$
}

Submitted: Mar 31, 2005; Accepted: Mar 15, 2006; Published: Mar 21, 2006

Mathematics Subject Classification: 05d05

\begin{abstract}
Let $0 \leq p \leq 1 / 2$ and let $\{0,1\}^{n}$ be endowed with the product measure $\mu_{p}$ defined by $\mu_{p}(x)=p^{|x|}(1-p)^{n-|x|}$, where $|x|=\sum x_{i}$. Let $I \subseteq\{0,1\}^{n}$ be an intersecting family, i.e. for every $x, y \in I$ there exists a coordinate $1 \leq i \leq n$ such that $x_{i}=y_{i}=1$. Then $\mu_{p}(I) \leq p$.

Our proof uses measure preserving homomorphisms between graphs.
\end{abstract}

One of the fundamental questions first studied in extremal graph theory is the question of bounding the size of an intersecting family of sets. The most basic theorem in this vein is the Erdős-Ko-Rado theorem ([2]) that states that if $k \leq n / 2$ and $F$ is an intersecting family of $k$-subsets of $\{1, \ldots, n\}$ then $|F| \leq\left(\begin{array}{l}n-1 \\ k-1\end{array}\right)$. The theorem we present here is the analogue of the EKR theorem in the setting of the discrete cube endowed with the product measure. This useful theorem, and several generalizations thereof has been proven and reproven in several papers (see e.g. [4], [5], [1], [3]), but curiously enough none of these proofs seem to be related to the one we present here which relies in a mysterious way on a decomposition of the $n$ dimensional torus into 1-dimensional circles.

Here is the main theorem:

Theorem 1.1 Let $0 \leq p \leq 1 / 2$ and let $\{0,1\}^{n}$ be endowed with the product measure $\mu_{p}$ defined by $\mu_{p}(x)=p^{|x|}(1-p)^{n-|x|}$, where $|x|=\sum x_{i}$. Let $I \subseteq\{0,1\}^{n}$ be an intersecting family, i.e. for every $x, y \in I$ there exists a coordinate $1 \leq i \leq n$ such that $x_{i}=y_{i}=1$. Then $\mu_{p}(I) \leq p$.

Before proving the theorem we must introduce some notation. All graphs $G$ considered in this note will come endowed with a probability measure $\mu_{G}$ defined on their vertex set.

\footnotetext{
${ }^{0}$ Key words and phrases: Intersecting families, Product measure.

${ }^{*}$ School of Computer Science and Engineering, Hebrew University, Jerusalem, Israel. email: dinuri at cs.huji.ac.il

†Institute of Mathematics, Hebrew University, Jerusalem, Israel. email: ehudf at math.huji.ac.il. Research supported in part by the Israel Science Foundation, grant no. 0329745.
} 


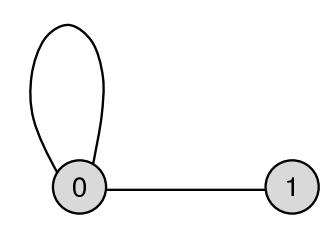

Figure 1: The Graph $G$

Given two graphs $H$ and $G$ a homomorphism between them is a mapping $\phi: V(H) \rightarrow$ $V(G)$ such that

$$
\{x, y\} \in E(H) \Rightarrow\{\phi(x), \phi(y)\} \in E(G) .
$$

Note that no restriction is imposed on the image of nonedges in $H$. Note also that the inverse image of an independent set in $G$ is an independent set in $H$. We will say that a homomorphism from $\phi: H \rightarrow G$ is measure preserving if for every $A \subseteq V(G)$

$$
\mu_{H}\left(\phi^{-1}(A)\right)=\mu_{G}(A) .
$$

We will write $H \rightarrow G$ if there exists a measure preserving homomorphism from $H$ to $G$. We will also need the notion of a weak graph product: given graphs $G_{1}, G_{2}$ their product $G_{1} \times G_{2}$ is defined as follows:

$$
V\left(G_{1} \times G_{2}\right)=V\left(G_{1}\right) \times V\left(G_{2}\right)
$$

and

$$
\left\{\left(u_{1}, u_{2}\right),\left(v_{1}, v_{2}\right)\right\} \in E\left(G_{1} \times G_{2}\right) \Leftrightarrow\left\{u_{i}, v_{i}\right\} \in E\left(G_{i}\right) \text { for } i=1,2 .
$$

We will write $G^{n}$ to denote the $n$-fold product of $G$ with itself, and always consider the product measure on the product of graphs. Note that if $H \rightarrow G$ via a homomorphism $\phi$ then $H^{n} \rightarrow G^{n}$ via the homomorphism $\phi^{n}$ which is just the application of $\phi$ coordinatewise.

For a graph $G$ define

$$
\bar{\alpha}(G)=\max \left\{\mu_{G}(I) \mid I \subseteq V(G), I \text { is an independent set }\right\} .
$$

Note that if $H \rightarrow G$ then $\bar{\alpha}(H) \geq \bar{\alpha}(G)$. Furthermore, since projection on a coordinate is a measure preserving homomorphism, $\bar{\alpha}\left(H^{n}\right) \geq \bar{\alpha}(H)$ for any graph $H$.

We now can present the proof of the theorem.

Proof: First note that if $p=1 / 2$ then $\mu_{p}$ is the uniform measure and the theorem is trivial because if a vector in $\{0,1\}^{n}$ belongs to $I$ then the antipodal vector to it cannot. Hence we shall assume $0<p<1 / 2$. Let us begin by defining two graphs, $G$ and $H$. $G$ is a graph on two vertices, $V(G)=\{0,1\}$. There is an edge between 0 and 1 , and also a loop from 0 to itself, as illustrated in Figure 1 . The measure $\mu_{G}$ is defined by $\mu(1)=p, \mu(0)=1-p$. It is not hard to see that $G^{n}$ is the non-intersection graph of $\{0,1\}^{n}$, i.e. that for $x, y \in\{0,1\}^{n}$

$$
\{x, y\} \notin E\left(G^{n}\right) \Leftrightarrow \exists 1 \leq i \leq n, x_{i}=y_{i}=1 .
$$

Furthermore due to the product measure on $G^{n}$ our theorem now translates precisely to the statement $\bar{\alpha}\left(G^{n}\right) \leq p$. 
Next consider the graph $H$ defined as follows. The vertex set of $H$ is the (continuous!) interval $[0,1]$ with the points 0 and 1 identified, i.e. the circle. There is an edge between two points $x$ and $y$ if the short arc between them on the circle is of length greater than $p$. (Note that had we not assumed $p<1 / 2$ then this graph would be empty.) We consider $H$ endowed with the uniform measure. It seems clear that the maximal measure of an independent set in this graph is obtained by an arc of length $p$, and indeed this is so.

Lemma $1.2 \bar{\alpha}(H)=p$.

Proof: Let $I \subseteq V(H)$ be an independent set of maximal measure. We may assume that $I$ is closed (replacing $I$ by its closure will not spoil independence.) Therefore we can find $x$ and $y$, two points in $I$ of maximal distance. Assume $\mathscr{x y}$ is the short $\operatorname{arc}$ between $x$ and $y$ on the circle. Consider the $\operatorname{arc} C=\stackrel{\curvearrowright}{a b}$ of length $1 / 2$ that contains the arc $\stackrel{\curvearrowright}{x y}$ such that the chord $a b$ is parallel to $x y$. A short reflection shows that replacing all points in $I$ that are not in $C$ by their antipodal points can only decrease the diameter of the set hence will not spoil the independence. Since $I$ could not have contained any pair of antipodal points this does not decrease the measure. Hence we may assume that $I$ is contained in $C$, and therefore in $\stackrel{x y}{y}$ and so the maximal possible value of $\mu_{H}(I)$ is obtained by an interval of length $p$.

A special property of $H$ that we will use is that $\bar{\alpha}\left(H^{n}\right) \leq \bar{\alpha}(H)$ for any $n \geq 1$. Recalling that the reverse inequality always holds, we have equality, $\bar{\alpha}\left(H^{n}\right)=p$. To see that indeed $\bar{\alpha}\left(H^{n}\right) \leq \bar{\alpha}(H)$ note that the vertex set of $H^{n}$, the $n$-dimensional torus, may be partitioned into sets of the form

$$
C_{x}=\{x+(t, t, \ldots, t) \mid t \in[0,1]\}
$$

where addition is done modulo 1 . It is not hard to see that each such set is a circle on which the induced graph, with its conditional measure, is isomorphic to $H$. Hence for any independent set $I \subseteq V\left(H^{n}\right)$ and any such set $C_{x}$ we have the 1-dimensional observation

$$
\mu_{C_{x}}\left(I \cap C_{x}\right) \leq p
$$

Integrating over all $x$ shows that $\mu_{H^{n}}(I) \leq p$ as required.

Finally we observe that $H \rightarrow G$ (take any mapping that maps an arc of length $p$ to 1 and the complement to 0 .) Hence

$$
\bar{\alpha}\left(G^{n}\right) \leq \bar{\alpha}\left(H^{n}\right)=\bar{\alpha}(H)=p .
$$

This completes the proof of the theorem. 


\section{References}

[1] I. Dinur, S. Safra, On the importance of being biased (1.36 hardness of approximating Vertex-Cover). Annals of Mathematics, to appear. Proc. of 34th STOC, 2002.

[2] P. Erdős, C. Ko, R. Rado, Intersection theorems for systems of finite sets, Quart. J. Math. Oxford, ser. 212 (1961), 313-318.

[3] E. Friedgut, On the measure of intersecting families, uniqueness and robustness, Submitted.

[4] P.C. Fishburn, P. Frankl, D. Freed, J.C. Lagarias, A.M. Odlyzko, Probabilities for intersecting systems and random subsets of finite sets, SIAM J. Algebraic Discrete Methods 7 (1986), no. 1, 73-79.

[5] P. Frankl and N. Tokushige, Weighted multiply intersecting families, Studia Sci. Math. Hungarica 40 (2003) 287-291. 